\title{
Fast Screening of Corrosion Trends in Metallic Glasses
}

Jingbei Liu ${ }^{1}$, Naijia Liu ${ }^{1}$, Meng Sun ${ }^{2}$, Jinyang Li ${ }^{3}$, Sungwoo Sohn ${ }^{1}$, Jan Schroers ${ }^{1, *}$

${ }^{1}$ Department of Mechanical Engineering and Materials Science, Yale University, New Haven, Connecticut, 06511, USA

${ }^{2}$ Department of Chemical and Environmental Engineering, Yale University, New Haven, Connecticut, 06520, USA

${ }^{3}$ School of Materials Science and Engineering, Southwest Jiaotong University, Chengdu, 610031, China

Corresponding author: jan.schroers@yale.edu

\section{Supporting materials}

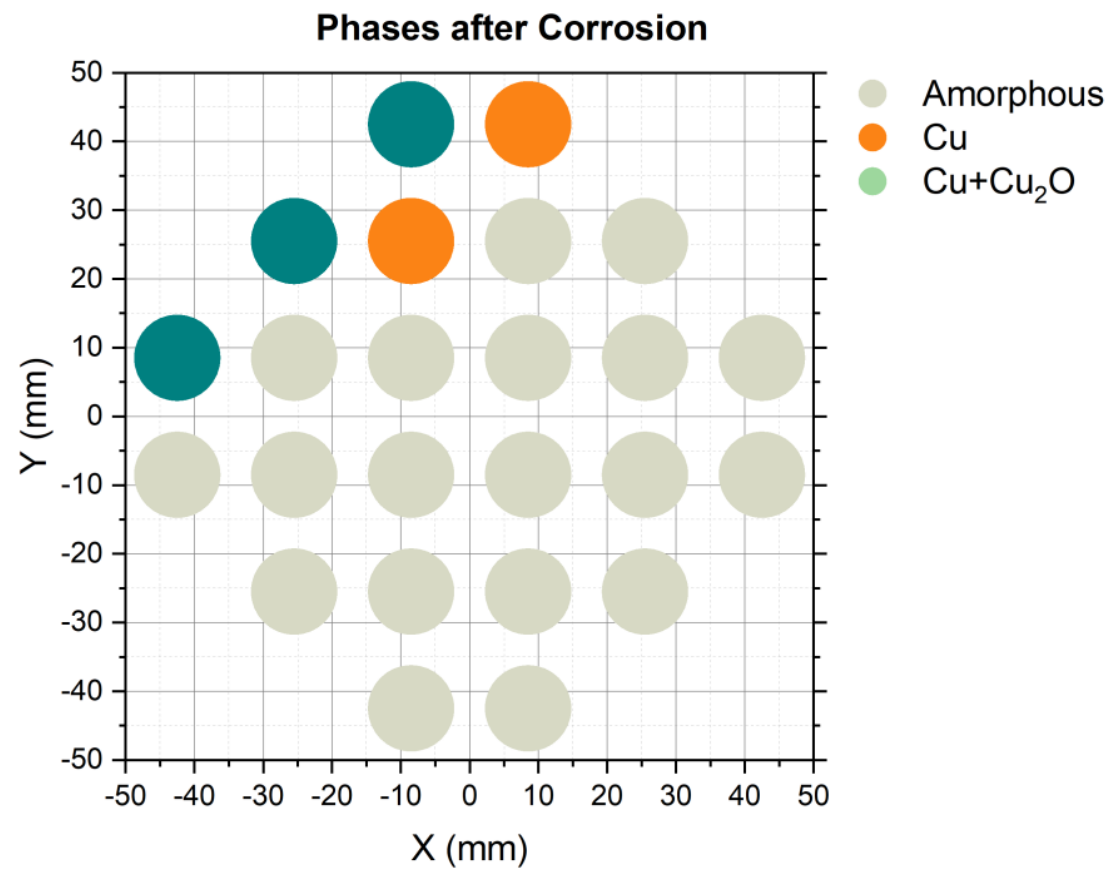

Figure S1. Phases presented of 24 thin film alloys after corrosion based on XRD spectrum with respect to their physical $x-y$ coordinates on the wafer. 


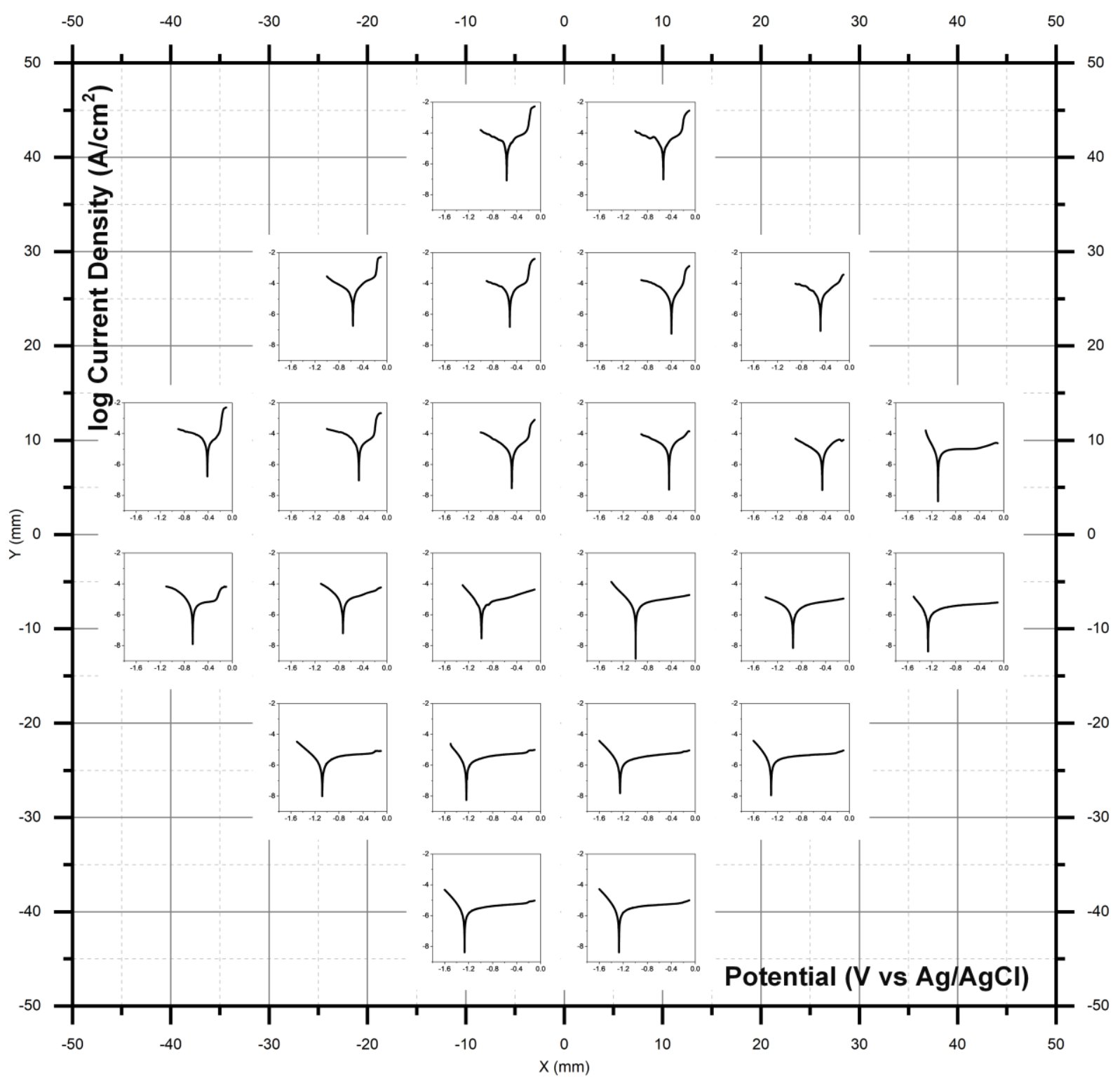

Figure S2. Polarization curves of 24 thin film alloys with respect to their physical x-y coordinates on the wafer. 


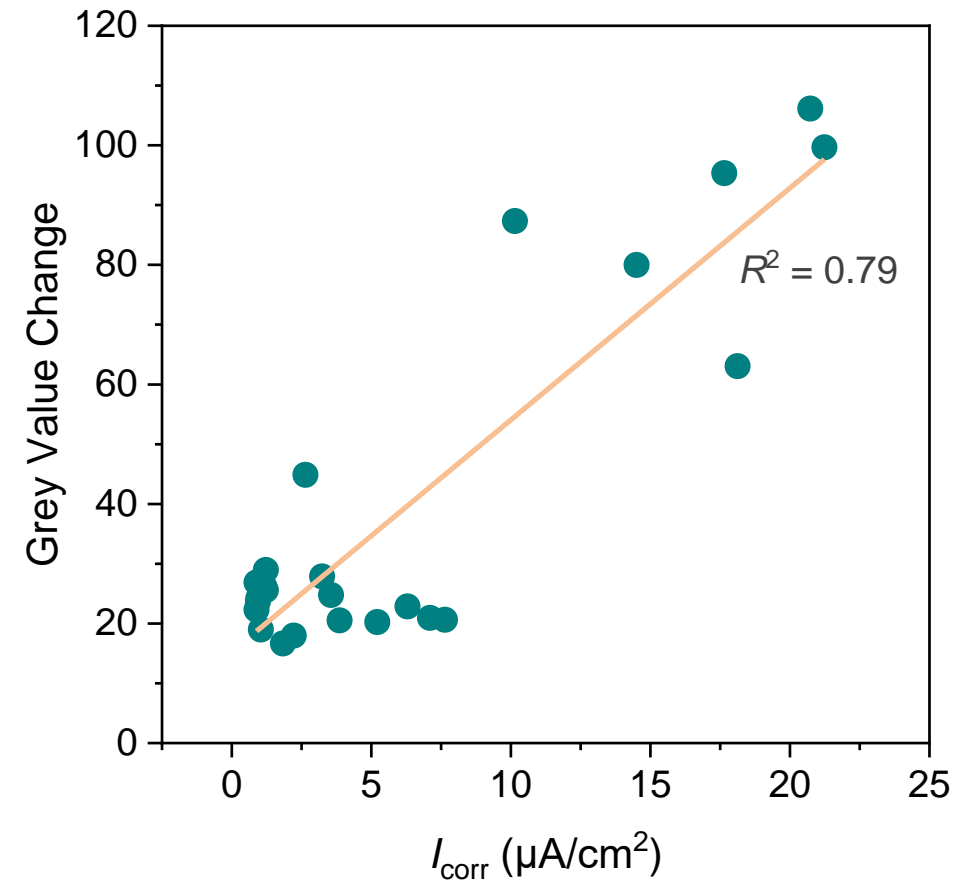

Figure S3. Correlation between corrosion current density $I_{\text {corr }}$ and grey value change of 24 thin film alloys. 